\title{
Representation Modeling Persona by using Ontologies: Vocabulary Persona
}

\author{
GAOU Salma ${ }^{1}$ \\ 1.Faculté des sciences . Université \\ Abdelmalek Essaadi. \\ Av Jbel Hayane Lot Oulianti N 7 \\ ETG 3, BP 565. Tétouan,MAROC.
}

\author{
EL KADIRI Kamal Eddine ${ }^{2}$ \\ 2. Faculté des sciences de Tétouan. \\ Université Abdelmalek Essaadi. \\ Mhannech II, B.P : 2121. \\ Tétouan,MAROC.
}

\author{
CORNELIU BURAGA Sabin ${ }^{3}$ \\ 3.Faculty of Computer Science. "A. \\ I. Cuza" University. \\ 16, G-ral Berthelot Street. Iasi, \\ 700483 ROMANIA.
}

\begin{abstract}
Semantic Web is then to add to all these resources semantics that allow computer systems to "understand" the meaning by accessing structured collections of information and inference rules that can be used to drive reasoning automated to better satisfy user requirements. Standard description of Web resources proposed by the $\mathrm{W3C}$, as the name implies, $\mathrm{RDF}$ (Resource Description Framework) is a meta-data used to guide the description of resources, to make it more "structured" information necessary for engines research and, more generally, to all necessary computer automated tool for analyzing web pages. The web is a new web sematique or all Web resources are described by metadata, which allows machines better use of these resources. Considering as a foundation specification FOAF (Friend Of A Friend), we use semantic structures (RDFa) to create an ontology and technologies in which it is implemented.Create a conceptual model (eg, an ontology) for personas and their uses in the context of human-computer interaction we will present some screenshots of execution of application.
\end{abstract}

Keywords-Semantic Web; FOAF; Persona; Vocabulary; Ontology; Persona;

\section{INTRODUCTION}

Semantic Web technologies and Semantic Web offers us a new approach to managing information and processes, the fundamental principle is the creation and use of semantic metadata. Using the semantics, we can improve the way information is presented. At its simplest, instead of providing a linear search results list, the results can be grouped by meaning. The use of semantic metadata is also crucial for the integration of information from heterogeneous sources, either within an organization or across organizations. [1]

For their implementation, management information, the integration of effective information and intergration of applications require that all information and underlying be semantically described and managed process, is that they are associated with a machine description of their meaning. This, the basic idea behind the Semantic Web has become very important to late 1990s [2] and in a more developed in the 2000s [3] form. The last half decade has seen intense activity in the development of these ideas, especially under the auspices of the World Wide Web Consortium (W3C) [4]. At the heart of all Semantic Web applications is the use of ontologies. A commonly accepted definition of an ontology is: "An ontology is an explicit and formal specification of a conceptualization of a domain of interest" [5] This definition focuses on two points, first, the conceptualization is formal and therefore allows reasoning by. the computer, and the ontology is designed to perform a particular area of interest. Ontology consists of concepts (also known as the class name), relationships (properties) and instances and axioms. Therefore, a brief definition was proposed as $<$ C,R,I,A $>$ 4-tuple, where C is a set of concepts, $\mathrm{R}$ a set of relations, $\mathrm{I}$ have a set of instances and A a set of axioms [6]. The first work in Europe and the United States on the definition of ontology languages has converged under the W3C to produce an OWL [7] Web Ontology Language. OWL provides mechanisms for the creation of all the elements of ontology concepts, instances, properties (or relations) and axioms [1]. OWL is based on the Resource Description Framework (RDF) [8], which is essentially a language for data modelling, also defined by the W3C. RDF is based on the graphs, but usually serialized as XML. Essentially, it's triplets: subject, predicate, object [1]. The Semantic Web is simply a web of data described and linked in order to establish the context or semantics that adhere to the defined grammar and language constructs [9]. In this article we try to create a conceptual model (eg, an ontology) for personas and their use in the context of human-computer interaction. These models are widely used in the design, development and science. They are powerful tools to represent the structures and relationships in order to better understand the complex, talk, or use. Without models, we have to make sense of raw unstructured data, without the benefit of an organizing principle. Good models emphasize the main characteristics of structures and relationships they represent and de-emphasize less important details [10].

\section{ONTOLOGIES}

Originally, ontology is a philosophical concept, in which philosophers have attempted to account for the existence of formal way. Semantic Web researchers have adapted this term in their own jargon and various definitions of ontology exist in computer literature.

However, the definition most referenced and also the plastic is that of [11] : $<$ ontology is an explicit formal specification of a conceptualization. > [14] emphasizes safe conceptual nature of an ontology made in order to share knowledge between humans and systems, and between systems. He considers conceptualization, sharing and reuse as the key concepts of an ontology. [15] defines an ontology as an explicit formal description of concepts in a domain of discourse (classes sometimes called concepts), properties of 
each concept describing the characteristics and attributes of the concept (attributes sometimes called roles or properties) and restrictions on the attributes (sometimes called facets restriction roles). Class concept and define the same term in what follows. A methodology for the development of an ontology is developed in [15].

In summary, computer science, an ontology is understood as a system of basic concepts that are related with each other and represented in a form understandable by a computer each.

The semantic web uses ontologies for various reasons. She uses a simple way to improve search relevance, the request expressed can only refer to a precise concept instead of using keywords ambiguous. More advanced applications using ontologies to combine information from a page of knowledge structures and inference rules.

Many computer languages have emerged to build and manipulate ontologies. In order to develop a standardized language, the $\mathrm{W} 3 \mathrm{C}$ has created the web group that has developed OWL. The acronym OWL includes both OIL specifications developed by the European community and Damil language developed by DARPA. OIL is used to define ontologies and DAML adds a few features to RDF Schema to make it easier to define new languages for communication between intelligent agents. OWL therefore defines a syntax for describing RDF vocabularies and build to create ontologies. However, OWL has three sub languages of increasing phrase designed for communities of developers and users that are specific:

- OWL Lite: is the OWL language in the easiest, it is intended to represent hierarchies of simple concepts.

- OWL DL: is more complex than the previous one, it is based on description logic as its name (OWL Description Logics). It is suitable for reasoning, and it guarantees the completeness and decidability of reasoning.

- OWL Full: is the most complex version of OWL, intended for situations when it is important to have a high level of description capability, even if they can not guarantee the completeness and decidability of calculations related to the ontology [12].

OWL ontologies are in the form of text files, documents OWL. The creation of an OWL document is subject to various recommendations [16].

Ontologies are formal and consensual specifications of conceptualizations that provide a shared understanding of a domain, an understanding that can be communicated across people and application systems [13]. This section shows the main components of an OWL ontology

Representation of semantics is necessary for the integration of systems, techniques that knowledge representation is used, it examines how to transform the expression of meaning in a formal representation manipulable by a machine, one way the most used is the ontology.
In this section, and based on [17] and [18] we will explain in detail each one of the above plus two components namely the axioms and instances:

- Concepts: A concept can be a physical object, a concept, an idea [19], the concepts are also called terms or class of ontology, are the objects manipulated by the base ontologies. They correspond to the relevant abstractions of the problem domain, selected according to the objectives that is given and the intended use of the ontology application. They are present in OWL by owl: Class.

- Relationship: translate the interactions between the concepts present in the domain analysis. These relationships are formally defined as any subset of a Cartesian product of $\mathrm{n}$ sets, that is to say $\mathrm{R}$ : $\mathrm{C} 1 \mathrm{x} \ldots \mathrm{x}$ $\mathrm{Cn} \quad \mathrm{C} 2 \mathrm{x}$ and include specialization relation (subsumption), the relationship of composition (meronymy), the instantiation relation, etc.. These relationships allow us to capture the structure and the interaction between concepts, which can represent a large part of the semantics of the ontology. They are presented in OWL by owl: ObjectProperty.

- Functions: are special cases of relationships in which the nth element of the relationship is uniquely defined from the n-1 preceding elements. Formally, functions are defined as follows: F: C1 C2 x ... x Cn-1 $\rightarrow \mathrm{Cn}$

- Axioms: can model always true assertions about abstractions in the field resulted in the ontology. They can combine concepts, relations and functions to define rules of inference which can be made, for example, the deduction, the definition of concepts and relationships, and then to restrict the values of the properties or arguments a relationship.

- Instances: or individuals constitute the extensional definition of ontology. They represent unique elements conveying knowledge about the problem domain. They are defined by OWL owl: Thing.

The engineering knowledge (IC) has long been considered the favorite field of development of expertise in system design based on knowledge [11]. Thus, the operating mechanism by inference, a type representation as declarative ontology, while following the inference rules defined in this ontology, is the source of the Intelligence system. The knowledge engineering has given rise to ontological engineering, where the ontology is the key object which must be addressed. The need for ontology and ontological engineering of knowledge-based systems beginning to be understood and accepted [20]. OWL ontologies are used to model domain knowledge [9]. 


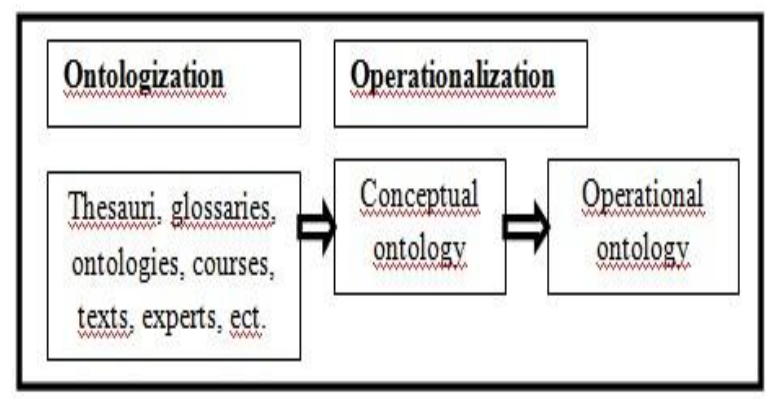

Fig. 1. Etapes de construction d'une ontologie

OWL provides a built-in class whose members correspond to modular parts of a semantic model. It is customary for the URI of an Ontology to correspond to the URL of the file on the Web where the ontology is stored[26]. OWL extends the expressivity of RDFS with additional modeling primitives. For example, OWL defines the primitives owl: equivalentClass and owl :equivalentProperty[27].

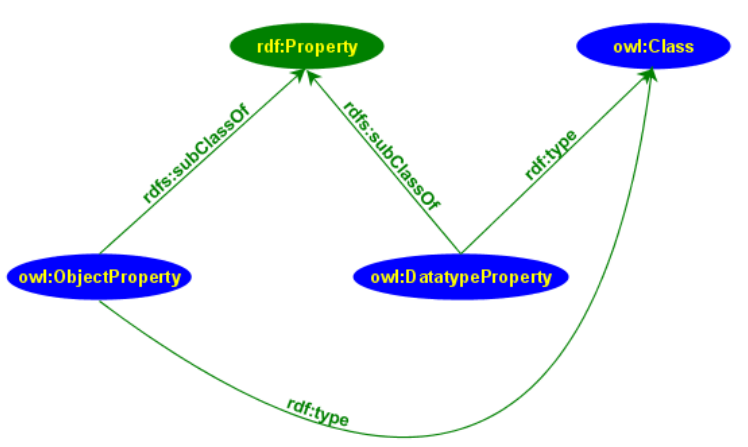

Fig. 2. OWL properties, classes, relations[21]

\section{FOAF VOCABULARY AND SyNTAX}

FOAF is not so much an application as ontology used by many applications. The Friend of a Friend project (FOAF) was one of the first to recognize the simple power of social networks. The FOAF project provides tools to connect people so a model that contains typical social attributes such as name, email address, interests, etc. [9]. The FOAF project is based on the use of machine readable Web homepages for individuals, groups, businesses and other types of things. For this, we use the "FOAF vocabulary" to provide a set of basic conditions that can be used in these web pages. At the heart of the FOAF project is a set of definitions to use a dictionary of terms that can be used to express claims about the world. The initial objective of FOAF has been on the description of the people, because people are the things that connect most of the other kinds of things that we describe in the Web: they are documents, attend meetings, are shown photos, and so on. FOAF vocabulary definitions presented here are written using a computer language (RDF / OWL) which makes it easy for software to handle some basic facts about the terms of the FOAF vocabulary, and consequently the things described in the FOAF document. FOAF document, unlike a traditional web page, can be combined with other materials to create a FOAF unified database of information [22].
Example[25]

Here is a very basic document describing a person:

\section{$<$ foaf:Person>}

<foaf:name>Dan Brickley</foaf:name>

<foaf:mbox_sha1sum>241021fb0e6289f92815fc210f9e9137262c252e</foaf: mbox_sha1sum>

<foaf:homepage rdf:resource="http://rdfweb.org/people/danbri/" />

$<$ foaf:img rdf:resource="http://rdfweb.org/people/danbri/mugshot/danbrismall.jpeg" / $>$

$<$ /foaf:Person>

FOAF is an application of the Resource Description Framework (RDF) because the area we describe - people - has so many competing needs a standalone size could not do them any justice. Using RDF, FOAF wins a powerful extensibility mechanism, allowing FOAF based descriptions can be mixed with claims made in any other RDF vocabulary [23].

FOAF, like the Internet itself, is an information system related. It is built using the Semantic Web technology decentralized, and was designed to allow the integration of data across a variety of applications, Web sites and services, and software systems. To achieve this, FOAF adopted a liberal approach to data exchange. It does not require you to say anything about yourself or others, or puts no limits on the things you say or the variety of the Semantic Web vocabularies that can be used to do this. The current specification provides a "dictionary" basic term about people and the things they do and do [24].

\section{PERSONA}

A persona is a typical user (the famous archetype), a fictional representation of target users, which can be used to set priorities and guide our design decisions interface [27].

The method is a technique personas Users centered design, initiated by Alan Cooper in 1999. This method can provide a common and shared vision of the users of a service or product, highlighting their goals, expectations and potential brakes, and offering a more engaging format. In the field of web persona is a fictional character who represents a targeted group. When designing a website, it may be necessary to define multiple personas that will represent each type of potential visitors. A good persona is not to stereotype users but to create users that seems real. That is why we have set goals and personality traits realistic. Based on the objectives of the persona and its specific characteristics (identity, age, familiarity with computers ...) you should check that the user interface to meet the needs of users represented by the personas [27].

Personas give us a precise way of thinking and communicating how users behave, how they think, what they want to accomplish and why. Personas are not real people, but they are based on the behaviors and motivations of real people that we have observed and represent them throughout the design process. They are composite archetypes based on behavioral data collected from many actual users encountered in the ethnographic interviews. Personas are based on patterns of behavior that we see in the research phase, so that we formalize in the modeling phase. Using personas, we can develop an understanding of the goals of our users in specific 
contexts - an essential tool for the use of user research to inform and justify our designs[28].

Personas are a model used to describe the objectives, skills, abilities, experience and technical context of the users. They are detailed descriptions of archetypal users built on understanding; very specific data models on real people. A character is not based on an individual - he is a construct developed through a detailed process, not the result of a search for the "right" (see the character creation for more details) . They are used by the design team (and largest project team) to describe and keep the foreground user (s) for which the system will be built [27].

Personas, like many powerful tools are a simple concept but must be applied with considerable sophistication. It is not enough to whip up a couple of profiles based on stereotypes and generalizations users; it is not particularly useful to include a photograph of a stock job title and call it a "persona." Personas to be effective tools for design, considerable rigor and finesse should be applied to the process of identification of significant and meaningful in user behavior trends and transform these into archetypes that represent a wide range of users [28].

\section{CATEGORIES AND PERSONAS CONSTRUCTION}

Persona is a technical approach to ensure the inclusion and optimizing the user experience in the design of an interactive medium. To be useful, the persona must come from real information about users without their creation which may be based on stereotypes. This approach allows one hand to filter and synthesize data users and secondly to unite all stakeholders around key profiles: the main tasks that should be an answer, user's needs and priorities appear more easily identifiable.

Categories of persona:

- Primary Persona

This persona is usually designated the primary persona. Indeed, each primary persona requires the presence of its own user interface in a particular application. Knowing that there will be more of a primary persona when their needs cannot be met by the same interface. The fewer the number of primary personas the better.

- Secondary Persona.

By focusing on the primary persona, the secondary persona's goals and needs can mostly be met. Nevertheless, there are a few needs specific to them that are not a priority for the primary persona. To meet the needs of a secondary persona, there may be small additions to the interface necessary. However, these additions should not negatively affect the experience of the primary persona.

\section{- Supplemental persona}

User persona which are neither primary nor secondary are called supplemental persona. The combination of primary and secondary personas represents completely supplemental persona's needs that are completely satisfied by the solution devised for one of our primaries.

- Customer persona
Customer personas match customer needs, as discussed by ((auteur) et al., (année)) and their treatment is similar to that of secondary personas.

\section{- Served persona}

Served persona some what differ from persona types discussed previously. Although they are directly affected by the use of the product, they are not users of the product at all.

- Negative persona

Negative persona they aren't users of the product, like served personas. They mediate between stakeholders and product team members by informing them that there are specific types of users that the product is not being built to serve [28].

The majority of studies that have been done on personas seem to focus on targets in the context of what distinguishes persona to another [29]. But with narrative perspective objectives are parts of what makes the persona act in a given situation. What difference personas are like in real life, personal characteristics possess persona (age, history, psyche, etc.).

Personas Construction

As a rounded character [30], the persona can be characterized by the following elements, namely:

- Body: body constitutes a human being. Sex, age, look helps the designer emphasise the Persona

- Psyche: to understand motivations for actions we need to understand what lies behind the motivation, the personality.

- Background : job position, family, education, socialand cultural positions explain motivations for actions.

- Emotional state : to know the emotional state furthers engagement in the Persona [31]. Inner needs and goals, ambitions and wishes create a foundation for the emotional state.

- Cacophony : two oppositional character traits [32]. The oppositional traits are what constitute the difference between a stereotype and a rounded character.

Persona is static but is dynamic when inserted into the actions of the scenario. In the scenario, the persona is in a context, in a specific situation with a specific purpose [33].

Persona Elements:

- Goals ,

- Attitudes (related to your context),

- Behaviors \& Tasks (in your context),

- Name,

- Photo,

- Tagline,

- Demographic Info (brief just to help "humanize" them), 
- Skill level,

- Environment,

- Scenarios (not all but perhaps the highest priority, most common or most telling about their needs) [34].

\section{The Persona Data Model And The Diagram}

As you can see, the persona model focuses on six categories: context, person, goals, image, organization and event.

The context class is extended by four sub-classes of society, the application of the contact points, which is linked to the address and location (country). Persona of each person must be identified by name, age and other demographic information. He has goals that are related to the application, and includes individuals, businesses and concrete targets. Each persona must have an image that is particularly a photograph that corresponds with the name. It should have affilation organization that provides knowledge and experience, are included education, training and expertise. This should not be limited only to the application. Persona must attend the event.

\section{A. Use case diagram}

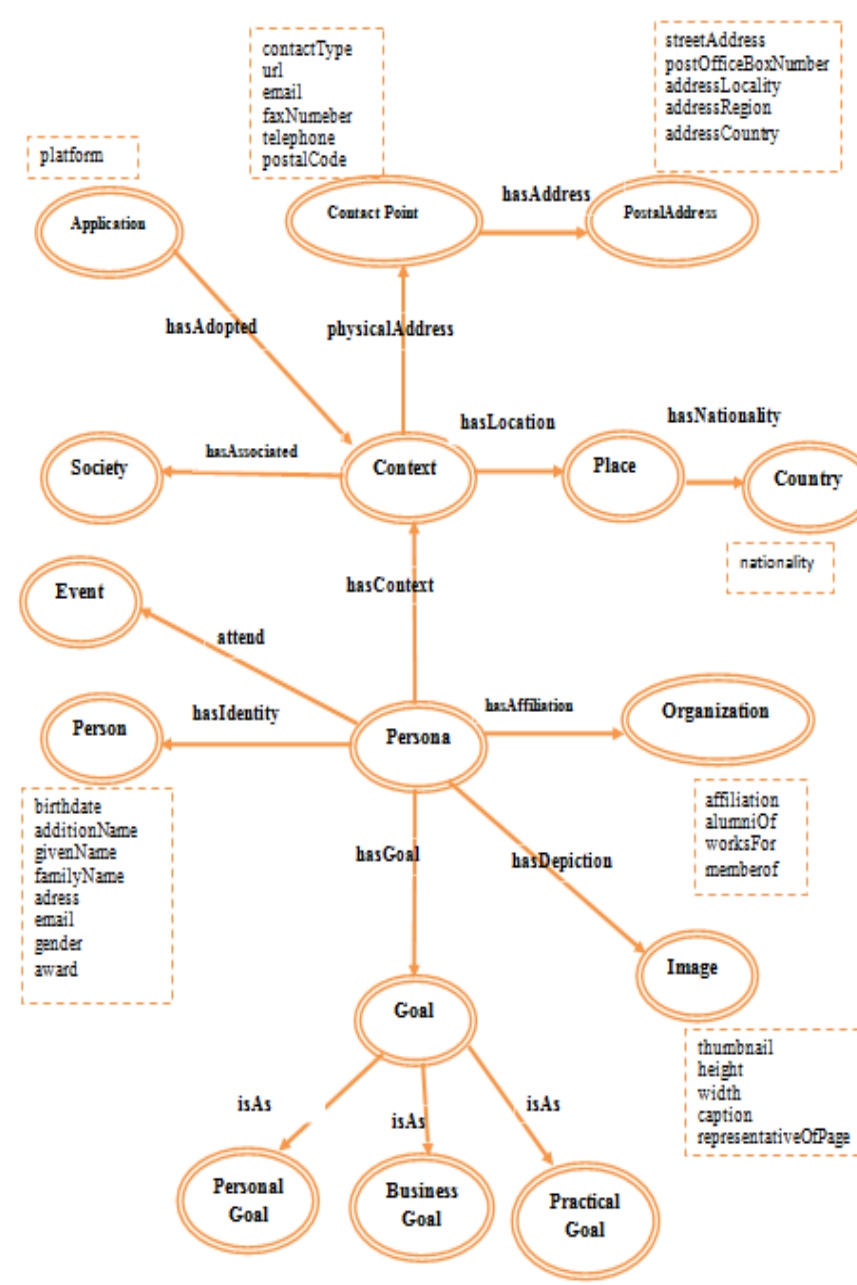

Fig. 3. Use case diagram of the Persona data model[35].

\section{B. Class diagram}

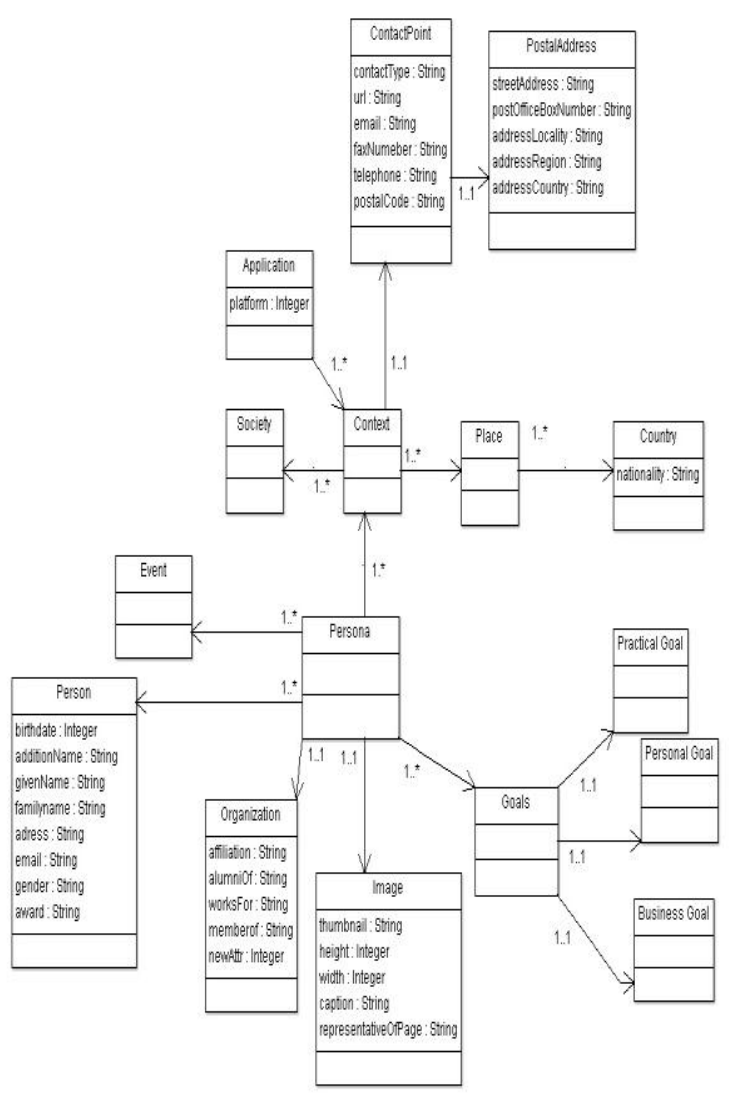

Fig. 4. Class diagram of the Persona data model.

TABLE I. THE TABLE BELOW SUMMARIZES ALL CLASS RELATIONS IN PERSONA.

\begin{tabular}{|l|l|l|}
\hline Subject Class & \multicolumn{1}{|c|}{ Object Class } & \multicolumn{1}{|c|}{ Definition } \\
\hline Persona & Person & $\begin{array}{l}\text { indicates that persona is } \\
\text { identified by demographic } \\
\text { information for person }\end{array}$ \\
\hline Persona & Context & $\begin{array}{l}\text { Indicates that Persona has } \\
\text { Context a context }\end{array}$ \\
\hline Persona & Goals & $\begin{array}{l}\text { Every Persona should have } \\
\text { Goals }\end{array}$ \\
\hline Persona & Image & $\begin{array}{l}\text { Every Persona should have } \\
\text { Image }\end{array}$ \\
\hline Persona & Organization & $\begin{array}{l}\text { Persona has affiliated to } \\
\text { Organizations }\end{array}$ \\
\hline Persona & Event & $\begin{array}{l}\text { Persona should attend the } \\
\text { Event }\end{array}$ \\
\hline Context & Place & Place of the location. \\
\hline Context & ContactPoint & $\begin{array}{l}\text { Indicates that Context has a } \\
\text { Contact point }\end{array}$ \\
\hline Application & Context & $\begin{array}{l}\text { Application has adopted } \\
\text { Context }\end{array}$ \\
\hline Context & Society & $\begin{array}{l}\text { Context has associated to } \\
\text { Society }\end{array}$ \\
\hline Contact Point & PostalAddress & ContactPoint has address in \\
\hline
\end{tabular}




\begin{tabular}{|l|l|l|}
\hline & & PostalAddress \\
\hline Place & Country & $\begin{array}{l}\text { Place has associated to Country } \\
\text { by nationality }\end{array}$ \\
\hline Goal & Practical Goal & $\begin{array}{l}\text { Indicates that Goal are } \\
\text { practical Goal efficiently }\end{array}$ \\
\hline Goal & Personal Goal & $\begin{array}{l}\text { Personal Goal should get an } \\
\text { adequate amount of work done, } \\
\text { be comfortable and fun }\end{array}$ \\
\hline Goal & Business Goal & $\begin{array}{l}\text { quality education is required for } \\
\text { Business Gola, for exemple }\end{array}$ \\
\hline
\end{tabular}

\section{PROTEGE}

As part of our modeling tool we have chosen for the creation of ontology is none other than Protege 2000 (Protégé200, 2008) because it allows viewing and comfortable and intuitive manipulation of concepts and relations up the ontology. Open source ontology editor, developed at the Department of Medical Informatics at Stanford University. The ontology editor "Protege" was used to edit the ontology of Persona with the aim to automatically generate the code for OWL, as well as for generating HTML documentation for our ontology. It should be noted that "Protege" offers very sure a lot of features, and we certainly did not all used. Protege also program or import a large number of "plugins" that can be downloaded directly from its official website. These plugins provide new features such as the ability to edit the ontology with different formats for describing ontology (RDF (S), OIL, DAML + OIL, OWL).

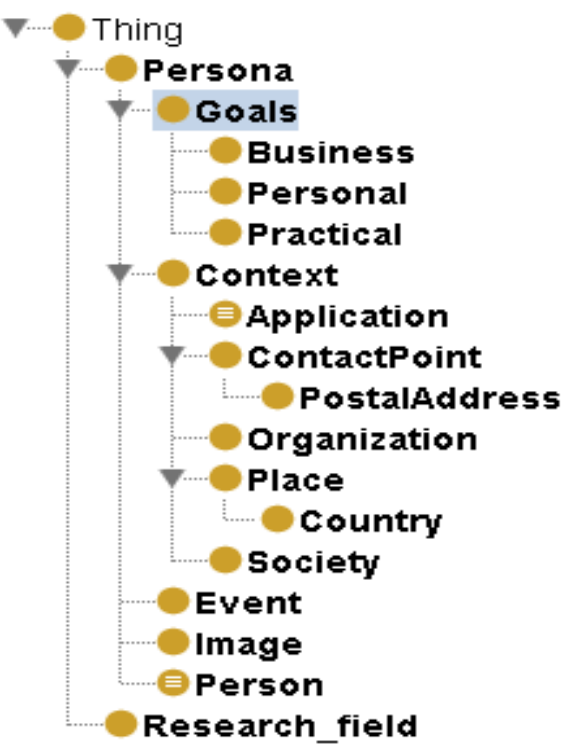

Fig. 5. Gives the list of classes in a hierarchical view.
A. $\quad$ This is the list of Data properties:

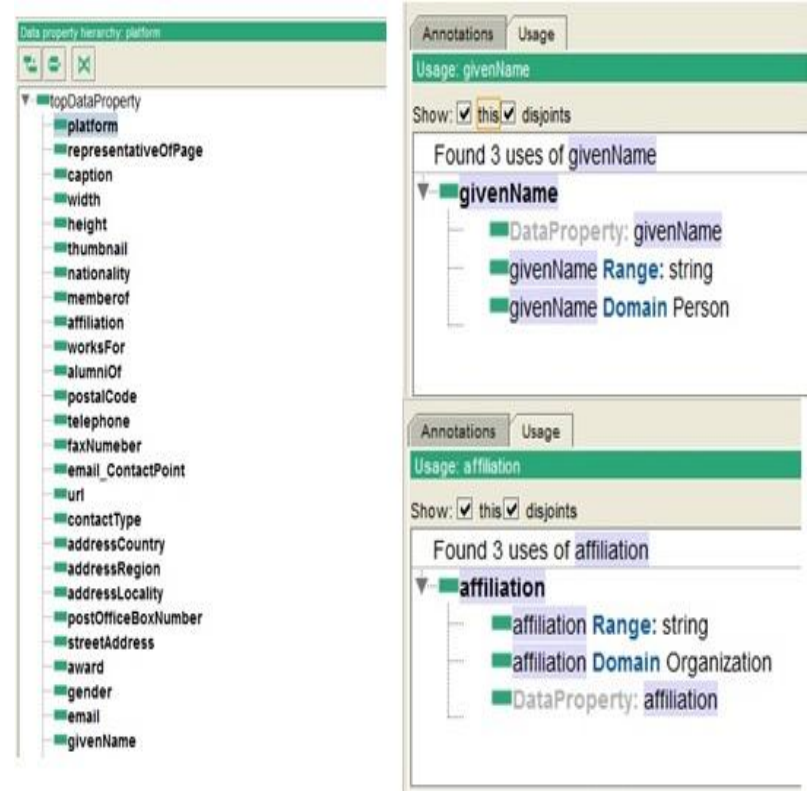

Fig. 6. Extract the Data property defined in the ontology Persona

B. This is the list of object properties:

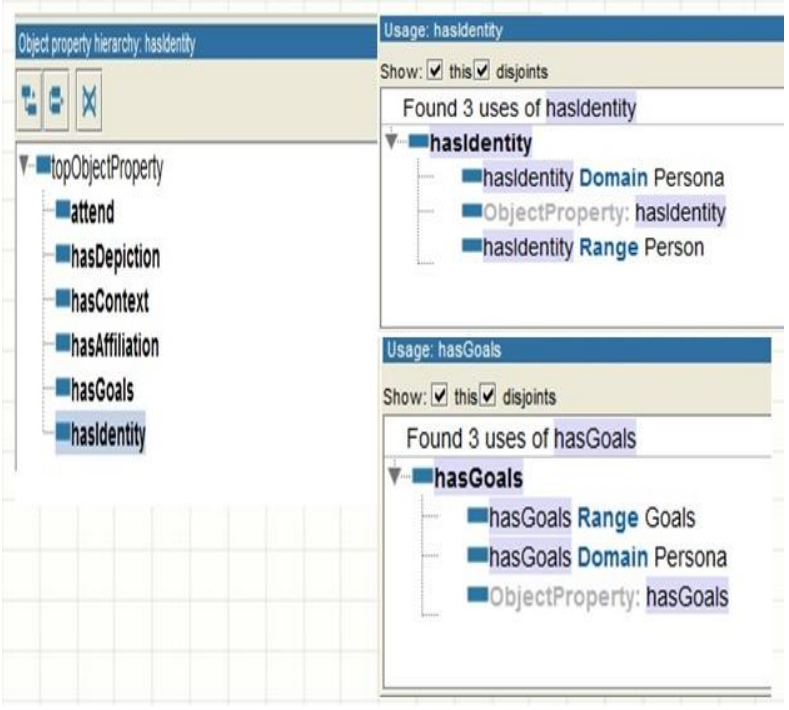

Fig. 7. Extract the Object property defined in the ontology Persona

\section{Strengths Of Personas As A Design Tool}

- Persona is a powerful design tool, multi-purpose, which helps to overcome several problems that currently plague the development of digital products. Personas help designers:

- Determine what a product should do and how it should behave. Persona goals and tasks form the basis of the design effort.

- Communicate with stakeholders, developers and other designers. Personas provide a common language for 
discussing design decisions and also help to keep the design centered on users at every stage of the process.

- Build consensus and commitment to the design. With a common language for a common understanding. Personas reduce the need for complex schematic models, it is easier to understand the many nuances of user behavior through the narrative structures that personas employ.

- Measure the effectiveness of the design. Design choices can be tested on personas in the same way that they can be presented to a real user during the training process. Although it does not replace the need to test with real users, it provides a powerful reality-check for designers trying to solve design problems. This allows design iteration to produce quickly and cheaply to the table, and the result is a much stronger base of design when it comes time to test with real people.

- Contribute to other product-related efforts such as marketing and sales plans. The authors have seen their customers repurpose personas within their organization, informing marketing campaigns, organizational structure, and other strategic planning activities. Business units outside of product development desire thorough knowledge of users of a product and see generally personas with great interest[33].

- You can use personas when you want:

- Making sense of research findings:To do this, you need to analyze the results and identify trends in research and capture the most important information about who they are, what they need to accomplish their skills, abilities and pain points, etc.

- Plan your product: To do this, analyze the competition through the eyes of your personas, features brainstorming possible using your personas and persona characteristics using a weighting matrix based on priorities.

- Explore design solutions: You need to prepare scenarios, mapping of the design, mood boards and explorations of visual design.

- Assess your solutions: You must make cognitive walkthroughs, design reviews with personas, user testing, user research underway with personas, Quality Assurance (QA) testing and bug bashes (focus quality assurance tests and create persona based on test cases, bug persona labelling.

- Supporting the release of your product: You will need to do the documentation, training, support materials (personas can help focus the teaching materials, guides, and editorial content), marketing and sales (tailor efforts based on personas, the distinction between users and customers (students compared, for example))

- Communicate with the project team and beyond: You have to share your learning with the rest of the team, gain consensus for designing early on ... before conception occurs, hang your personas around the room to keep the project development and create a common language and a shared vision [36].

\section{Deploying The Application PeRsona}

Persona web application developed using JSP and Servlet technologies. To be functional, it must be deployed in an HTTP application server with JSP / Servlet container. Apache Tomcat is both HTTP server (Apache) and Servlet / JSP container, which makes it an ideal candidate for the deployment of our application. The figure below shows the deployment scheme of Persona application.

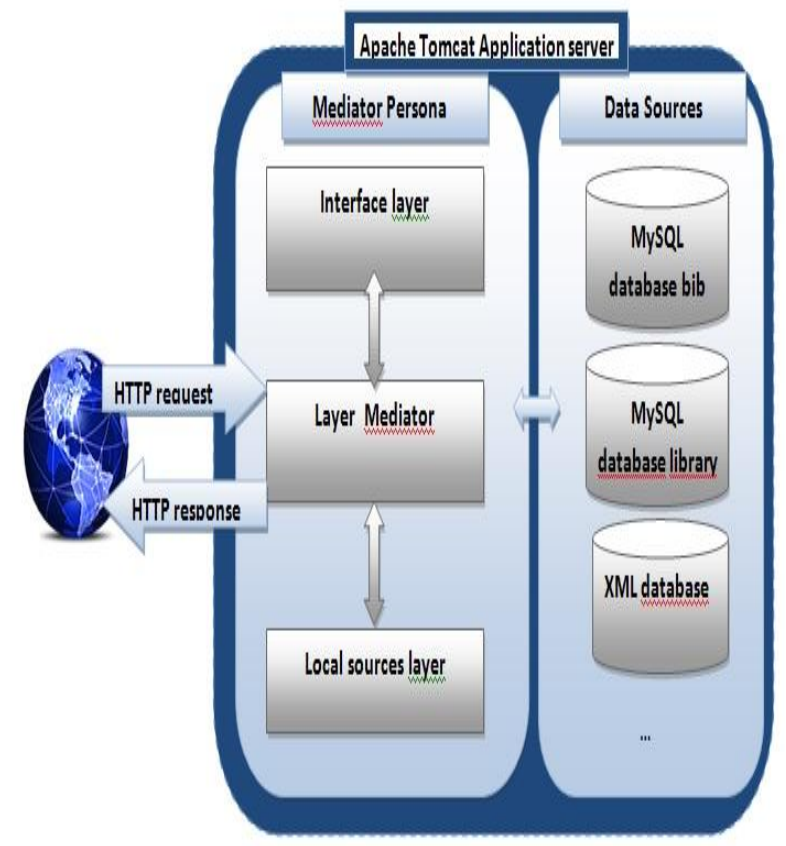

Fig. 8. Diagram of deployment Persona Application

The deployment of the application on Apache Tomcat persona is done by placing the directory containing the application files in the webapps directory of Tomcat.

\section{Images Of APPlicAtion EXECUTION}

In what follows we will present some screenshots of execution of our application, the homepage is the first window website, where you can access the different menus of the site persona. It contains five main menus (Identity, Organization, Context, Event, and Goals).

- Context menu contains three submenus (Place, Society and Contact point).

- Goals menu contains three submenus (Personal, Business and Practical).

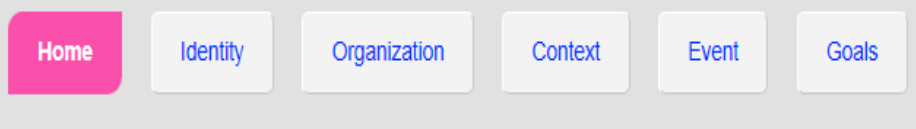

Fig. 9. Menus of the site persona. 


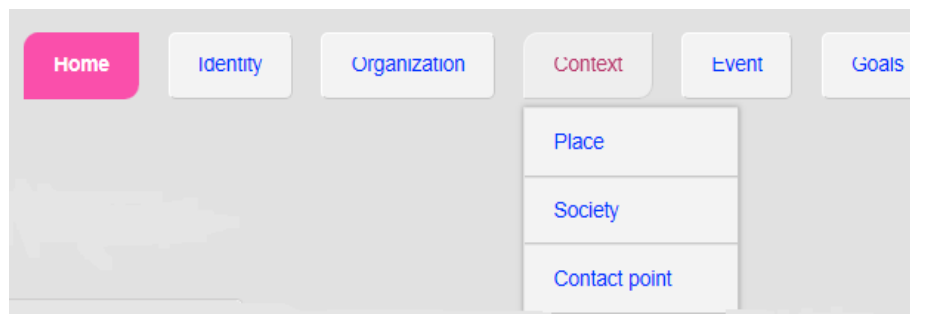

Fig. 10. Context menus active with submenus.

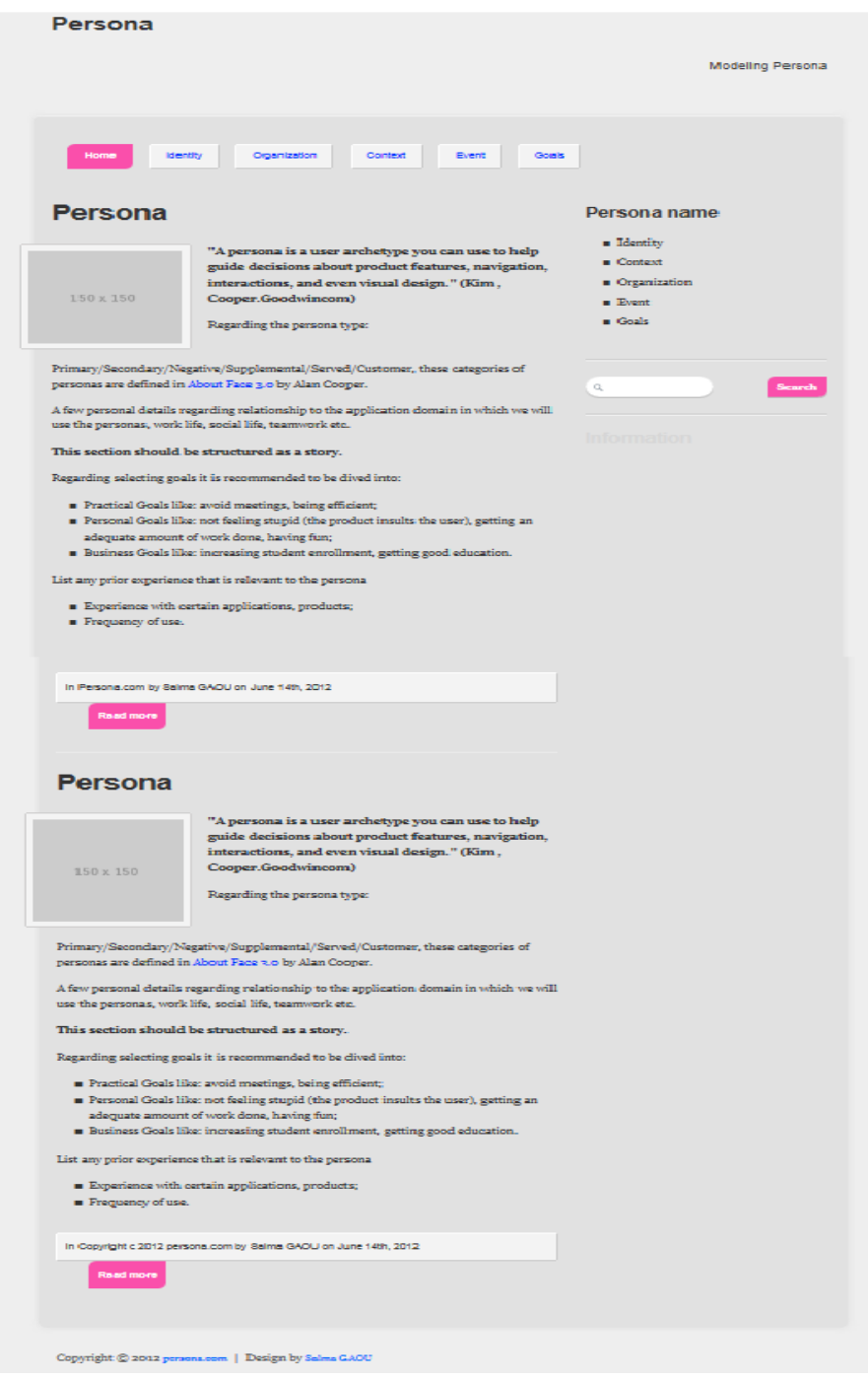

Fig. 11. the first page of website persona

To access this page below the identity, just click on the menu Identity This file contains all information (family name...) for each persona, also includes many features including:

The addition, modification and removal identity.

The same think for context (place, Society and Contact point), event, goals (Personal, Business and Practical), organization.
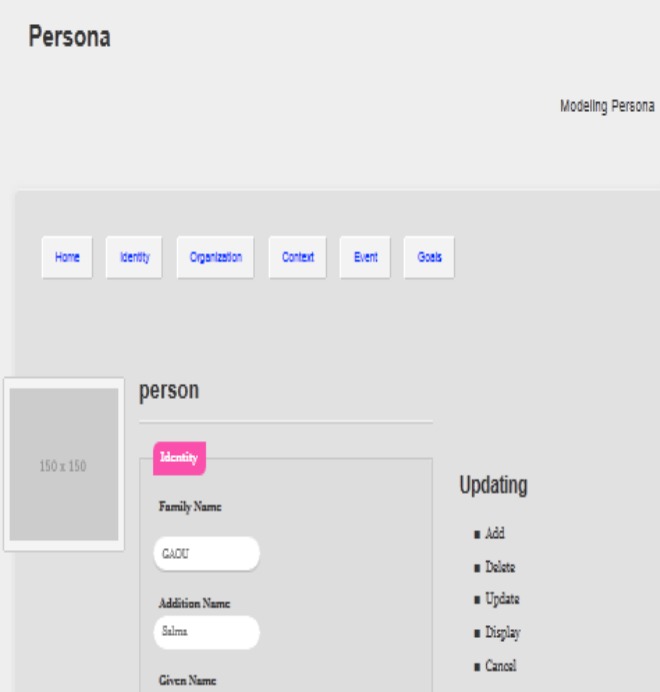

Copyight Q 2012 promenn | Design by sims CuOC

Fig. 12. page Identity of website persona

\section{CONCLUSIONS}

In this article, we introduced the notion of ontology and several methods and tools for ontology engineering. In our study, taking into consideration the characteristics and benefits of ontologies, we focused on the ontology for building a persona vocabulary.

Like many powerful tools, personas are a simple concept but must be applied with considerable sophistication. It is not enough for some profiles based on stereotypes and generalizations users, it is not particularly useful to attach photos to a job title and call it a "persona." Personas to be effective tools for design, rigor and considerable finesse should be applied to the process of identification of significant and meaningful in user behavior trends and transform these into archetypes that represent a wide range of users.

In addition, ontologies have the potential to allow a true knowledge sharing and reuse of heterogeneous agents, both human and computer. A major challenge is still open alignment 
of different ontologies to provide interoperability between heterogeneous agents. Considering that we were able to achieve the objectives of the party in this document, and we made the right choices about the tools for implementation, so that our work will be a great way for other future projects.

Looking ahead, we plan to expand our study using another technique, namely: first, the development of other ontologies and combine with ours to enrich the vocabulary used for annotating and research. Then test the possibility of reasoning provided by OWL. Finally, our next task will be presented the implementation details of the application, and is going to use for the realization of our application: Development Tool: JBuilder7, Virtual Machine, Java: J2SDK 1.4.2, Web Server JSP Tomcat 4.x, Libraries: Jena version 2.3 and Servlet / JSP Version 2.3/1.2 (included in Tomcat 4.x).

\section{ACKNOWLEDGMENTS}

Research reported in this paper has been partially financed by doctoral research within the Faculty of Computer Science University, Alexandru Ioan Cuza of Iasi in the program $<<$ Eugen Ionescu >> 2011-2012, and thank you for Dr. SabinCorneliu Buraga Associate Professor Faculty of Computer Science in Iasi for helping.

\section{References}

[1] Semantic Web Technologies, trends and research in ontology- based systems,John Davies, BT, UK,Rudi Studer,University of Karlsruhe,Germany,Paul Warren,BT, UK

[2] Berners-Lee T.1999. Weaving the web. Orion Busines Books.

[3] Berners-LeeT , Hendler J, Lassila O.2001.The semantic web. In Scientific American, May 2001.

[4] W3C staff assigned to the W3C Semantic Web Activityare Sandro Hawke (see also private blog ), Ivan Herman (see also private blog ), and Eric Prud'hommeaux , Ivan Herman, Semantic Web Activity Lead, 2011-11-07. en ligne : http://www.w3.org/2001/sw

[5] Gruber T.1993.A translation approach to portable ontologie. Knowledge Acquisition 5(2): 199-220, http://Kslweb.stanford.edu/KSL_Abstracts/KSL-92-71.html

[6] Staab S, Stuber R (Eds). 2004. Handbook on ontologies. International Handbooks on Information Systems. Springer : ISBN 3-540-40834-7.

[7] Ivan Herman 6 September 2007: W3C Launches the OWL Working Group, en ligne : http://www.w3.org/2004/OWL/

[8] 10 February 2004 , RDF is a standard model for data interchange on the Web. In ligne : http://www.w3.org/RDF/

[9] Semantic Web Programming,John Hebeler,Matthew Fisher,Ryan Blace,Andrew Perez-Lopez,septembrie 2009

[10] About Face 3 The Essentials of Interaction Design Alan Cooper, Robert Reimann, and Dave Cronin, 2007.

[11] Tim Berners-Lee, James Hendler, Ora Lassila The Semantic Web, Scientific American, May 2001

[12] Thomas R.Gruber. Ontolingua: A mechanism to mobile ontologies. Knowledge Systems Laboratory Technical Report KSL-91-66, Stanford University, version 3.0, CA, 1992.

[13] Xavier Lacot (2005) Introduction à OWL, un langage XML d'ontologies Web.
[14] Personas Added by Gary Thompson, last edited by Daphne Ogle on May 25, 2010.

[15] Riichiro Mizoguchi. The role of ontological engineering in the field of ILE

[16] Natalya F.Noy and Deborah L.McGuinness. Ontology development 101: A guide to creating your first ontology.

[17] Michael k.Smith, Chris Welty, Deborah L.McGuinness. OWL Web Ontology Language-Reference. http://www.w3.org/TR/2004/REC-owlref-20040210/ (online June 16, 2005).

[18] Tr.Gruber:. A translation approach to mobile ontology specification. Knowledge Acquisition 5 (2): pp. 199-220, 1993.

[19] Gomez-Perez. Recent developments in matters of design, maintenance and use of ontologies. 3emes meetings TIA Terminology and Artificial Intelligence, Vol 19, pp. 9-20, 2000.

[20] Sticef.org (2003) Apport de l'ingénierie ontologique aux environnements de formation à distance

[21] ATHENA WP4 SKOS Workshop Rome, ICCU, 16-17 July 2009 in ligne : http://www.w3c.it/talks/2009/athena/slides.html\#(52)

[22] D-FOAF - SECURITY ASPECTS IN DISTRIBUTED USER MANAGEMENT SYSTEM, Slawomir Grzonkowski, Adam Gzella, Henryk Krawczyk, Sebastian Ryszard Kruk, Francisco J. MartinRecuerda Moyano, Tomasz Woroniecki , Gdansk University of Technology, ul. Narutowicza 11/12, 80-952 Gdansk, Poland,

[23] Dan Brickley, Libby Miller.The Friend of a Friend (FOAF) project, Edd Dumbill's writings.2000, http://www.foaf-project.org/about.

[24] Dan Brickley, Libby Miller. FOAF Vocabulary Specification 0.98. Namespace Document 9 August 2010 - Marco Polo Edition. Creative Commons Attribution License. http://xmlns.com/foaf/spec/.

[25] Semantic Web for the Working Ontologist Effective Modeling in RDFS and OWL, Second Edition, Dean Allemang,Jim Hendler,2006.

[26] Linked Data Evolving the Web into a Global Data Space,Tom Heath,Christian Bizer, 2011.

[27] PERSONAS: définition et démarche. J.C. GROSJEAN. Quality Street, Coaching Agile, Management Agile \& Lean, Expérience Utilisateur, Tests. 29 décembre 2008

[28] About Face 3 The Essentials of Interaction Design Alan Cooper, Robert Reimann, and Dave Cronin, 2007.

[29] Pruitt, J. and J. Grudin (2003). Personas: Theory and Practice. 2003.

[30] Nielsen, L. (2002). From User to Character. DIS2002, London.

[31] Smith, M. (1995). Engaging Characters: fiction, emotion, and the cinema, Clarendon Press.

[32] Horton, A. (1999). Writing the Character-Centered Screenplay. L.A., University of California Press.

[33] Proceedings of the Third Danish Human-Computer Interaction Research Symposium,Morten Hertzum, Computer Science, Roskilde University ,Simon Heilesen, Communication Studies, Roskilde University ,Writings in Computer Science, No. 98 ,Roskilde University, Roskilde, Denmark, 2003.

[34] Persona Creation Added by Daphne Ogle, last edited by Allison Bloodworth on May 26, 2009.

[35] S. Gaou, K. E. El Kadiri, and S.CORNELIU BURAGA. Journal International Journal of Computer Science Information and Engineering Technologies l'article $<<$ USE OF ONTOLOGIES IN MODELING PERSONA $\gg$.

[36] Personas Added by Gary Thompson, last edited by Daphne Ogle on May 25, 2010. 